\title{
Histone deacetylase 5 promotes the proliferation and invasion of lung cancer cells
}

\author{
LOU ZHONG $^{1 *}$, SIYUAN SUN $^{2 *}$, SUMEI YAO $^{3}$, XIAO HAN $^{1}$, MINGMING GU $^{1}$ and JIAHAI SHI ${ }^{1}$ \\ ${ }^{1}$ Department of Thoracic Surgery, Affiliated Hospital of Nantong University; \\ ${ }^{2}$ Department of Clinical Medicine, Nantong University Xinglin College; \\ ${ }^{3}$ Department of Respiratory Medicine, The Second Affiliated Hospital of Nantong University, \\ Nantong, Jiangsu 226001, P.R. China
}

Received November 23, 2017; Accepted July 13, 2018

DOI: $10.3892 /$ or.2018.6591

\begin{abstract}
Histone deacetylase 5 (HDAC5), as a member of the class IIa family of HDACs, is frequently dysregulated in human malignancies. However, little is known regarding the specific role of HDAC5 in lung cancer. We aimed to evaluate HDAC5 expression in human lung cancer and to determine the effects of HDAC5 on lung cancer cells. First, the expression levels of both HDAC5 protein and mRNA were evaluated in lung cancer tissues and cell lines by western blot analysis and RT-qPCR, and the results suggested that HDAC5 was significantly upregulated in human lung cancer tissues and cell lines. To address the effects of HDAC5 on the biological behavior of human lung adenocarcinoma cells, we generated human lung cancer A549 cell lines in which HDAC5 was either overexpressed or depleted. The results indicated that overexpression of HDAC5 significantly promoted the proliferation and invasion, and inhibited the apoptosis of A549 cells. On the contrary, HDAC5 knockdown largely decreased the proliferation and invasion and enhanced the apoptosis of A549 cells. Furthermore, we demonstrated that HDAC5 overexpression promoted the expression of DLL4, Six1, Notch 1 and Twist 1 in A549 cells. Downregulation of HDAC5 caused a significant inhibition of the expression of DLL4, Six1, Notch 1 and Twist 1 in A549 cells. Taken together, our data demonstrated that HDAC5 displayed a significant upregulation in lung cancer, and elevated HDAC5 might be involved in the potentiation of proliferation and invasion of lung cancer cells, as well as the inhibition of lung cancer cell apoptosis by the upregulation of DLL4, Six1, Notch 1 and Twist 1. The present
\end{abstract}

Correspondence to: Professor Jiahai Shi, Department of Thoracic Surgery, Affiliated Hospital of Nantong University, 20 Xishi Road, Nantong, Jiangsu 226001, P.R. China

E-mail: zhonglount@163.com

${ }^{*}$ Contributed equally

Key words: HDAC5, lung cancer, proliferation, apoptosis, invasion study may provide an evidence for the potential application of HDAC5 inhibitors in the therapy of lung cancer.

\section{Introduction}

Lung cancer is a debilitating neoplasm, and accounts for significant morbidity and mortality worldwide (1). Despite significant progress seen in the last decade in regards to treatment regimens including surgery, radiotherapy and chemotherapy, and ongoing research development, the survival rate of patients with lung cancer is still less than satisfactory. A substantial number of patients succumb to the disease within the weeks following diagnosis, and the 5-year survival rate does not exceed $15 \%$ (2-5). The poor prognosis of lung cancer mainly results from its high degree of malignancy (malignant proliferation, invasion and migration). Most patients with lung cancer do not die of primary cancer, but rather die of metastatic cancer $(6,7)$. Therefore, the key molecules that mediate lung cancer metastasis have become a focus of scientific research.

The histone deacetylases (HDACs) form a family of enzymes, which have fundamental roles in the epigenetic regulation of gene expression and contribute to proliferation, differentiation, apoptosis and cell cycle progression (8-10). HDACs are frequently dysregulated in human malignancies and have therefore become therapeutic targets in cancer therapy (11). As a member of the class IIa family of HDACs, histone deacetylase 5 (HDAC5) is known to undergo nuclear-cytoplasmic shuttling and to be a prominent regulator of cellular and epigenetic processes that underlie the progression of human disease, including cardiac diseases and tumorigenesis (12-14). A growing body of literature suggests that HDAC5 is extensively expressed in many cancers. Li et al demonstrated that HDAC5 was extensively expressed in human breast cancer tissues, and high HDAC5 expression was associated with poor patient prognosis. Downregulation of HDAC5 was found to suppress breast cancer cell proliferation, invasion and migration, and promote breast cancer cell apoptosis (15). He et al showed that HDAC5 was upregulated in human colorectal cancer. Overexpression of HDAC5 significantly improved the proliferation of colorectal cancer cells. On the contrary, HDAC5 knockdown was found to suppress colorectal tumor cell growth (8). Feng et al showed that 
HDAC5 was increased in human hepatocellular carcinoma. Overexpression of HDAC5 promoted liver cancer cell proliferation, and inhibition of HDAC5 significantly inhibited liver cancer cell proliferation (16). Chen et al found that HDAC5 was upregulated in osteosarcoma, and overexpression of HDAC5 promoted the proliferation of osteosarcoma cells. In contrast, HDAC5 knockdown inhibited the proliferation of osteosarcoma cells (9). Liu et al demonstrated that HDAC5 displayed high expression in melanoma cells compared with normal skin cells. HDAC5 knockdown was found to suppress the proliferation and metastasis of melanoma cells (17). Milde et al found that HDAC5 displayed a significant upregulation in high-risk medulloblastoma compared with low-risk medulloblastoma, and the upregulation of HDAC5 was associated with poor patient survival (18). In summary, HDAC5 has been found to play an important role in tumorigenesis, metastasis and invasion (16-18). However, little is known regarding the specific role of HDAC5 in lung cancer.

In the present study, lung cancer cell lines (A549, HCC827 and 95-D) and human bronchial epithelial cells (HBE) were used to detect the expression of HDAC5 by western blotting and RT-qPCR. The effects of HDAC5 on A549 cell proliferation, apoptosis and invasion were assayed. In addition, we analyzed the effects of HDAC5 on the expression of proteins, DLL4 (Delta-like 4), Six1, Notch 1 and Twist 1 in A549 cells. These data may provide information for the prediction of lung cancer prognosis and the establishment of targeted therapies.

\section{Materials and methods}

Specimens. The present study was reviewed and approved by the Ethics Committee of the Affiliated Hospital of Nantong University. All patients volunteered to participate in the study and signed a written informed consent. Fresh lung cancer tissues and matched adjacent non-tumor tissues were collected from 18 non-small cell lung cancer (NSCLC) patients that underwent surgical resection at the Affiliated Hospital of Nantong University from July 2015 to January 2017. The median patient age was 61 years (range, 48-72 years) and 13 patients $(72.2 \%)$ were male. Before surgery, all the patients received no radiotherapy and chemotherapy, and had no other treatment history, nor presented with inflammatory diseases. All tissue specimens collected from patients with NSCLC were immediately frozen in liquid nitrogen upon surgery, and were transported to the laboratory and stored at $-80^{\circ} \mathrm{C}$ for further tissue preparation.

Materials. All cell culture reagents were obtained from Gibco/Thermo Fisher Scientific, Inc. (Waltham, MA, USA). Human lung cancer cell lines (A549, HCC827 and 95-D) and human bronchial epithelial (HBE) cells (https://www.atcc. org/Products/All/PCS-300-010.aspx) were purchased from the American Type Culture Collection (ATCC; Manassas, VA, USA). Protein extraction buffer, MTT reagent, BCA protein concentration assay kit, Annexin V-FITC and propidium iodide (PI) were purchased from Beyotime Institute of Biotechnology (Haimen, China). Polyvinylidene difluoride (PVDF) membranes were supplied by Millipore (Bedford, MA, USA). Pierce ECL chemiluminescence detection kit was obtained from Thermo Fisher Scientific, Inc. Transwell inva- sion chamber was supplied by Costar Corp. (Cambridge, MA, USA). Matrigel was purchased from Collaborative Biomedical Products (Bedford, MA, USA). The antibodies used in this study included rabbit anti-HDAC5 polyclonal antibody (Abcam, Cambridge, UK; cat. no. ab55403), rabbit anti-delta-like 4 (DLL4) antibody (Cell Signaling Technology, Inc., Danvers, MA, USA; cat. no. 2589T), rabbit anti-SIX homeobox 1 (SIX1) antibody (LifeSpan BioSciences, Seattle, WA, USA; cat. no. LS-C490560-100), mouse anti-Notch 1 monoclonal antibody (Invitrogen Antibodies/Thermo Fisher Scientific, Inc.; cat. no. MA1-81888), rabbit anti-Twist 1 polyclonal antibody (Cell Signaling Technology, Inc.; cat. no. 46702S), mouse anti- $\beta$-actin monoclonal antibody (R\&D Systems, Minneapolis, MN, USA; cat. no. MAB8929), horseradish peroxidase-conjugated goat anti-rabbit (cat. no. 31239) and goat anti-mouse (cat. no. 31185) IgG polyclonal antibodies (Invitrogen Antibodies/Thermo Fisher Scientific, Inc.).

Cell culture and treatment. HBE cells and A549, HCC827 and 95-D cells were all cultured in RPMI-1640 medium containing $10 \%$ fetal bovine serum (FBS), $2 \mathrm{mM}$ L-glutamine, $1 \mathrm{mM}$ sodium pyruvate, $10 \mathrm{mM}$ HEPES, $1.5 \mathrm{~g} / \mathrm{l}$ sodium bicarbonate, $4.5 \mathrm{~g} / \mathrm{l}$ glucose, $50 \mathrm{U} / \mathrm{ml}$ penicillin and $50 \mu \mathrm{g} / \mathrm{ml}$ streptomycin at $37^{\circ} \mathrm{C}$ in a humidified atmosphere with $5 \% \mathrm{CO}_{2}$. When cells reached $70-80 \%$ confluence, trypsin digestion was performed for passage. Cells in logarithmic growth phase were digested with $0.25 \%$ trypsin and collected for further experiments.

A549 cells were chosen to perform further experiments. A549 cells $\left(2 \times 10^{5}\right)$ were seeded in a 6 -well tissue culture plate with $2 \mathrm{ml}$ antibiotic-free RPMI-1640 medium supplemented with $10 \%$ FBS. When cells reached $60-80 \%$ confluence, the cells were transfected with HDAC5 siRNA, pcDNA3.1-HDAC5 and control vector (Guangzhou RiboBio Co., Ltd., Guangzhou, China) using Invitrogen ${ }^{\mathrm{TM}}$ Lipofectamine $^{\circledR} 2000$ (Thermo Fisher Scientific, Inc.) according to the manufacturer's specification. Then, A549 cells were incubated with the compound at $37^{\circ} \mathrm{C}$ in a $\mathrm{CO}_{2}$ incubator for $5 \mathrm{~h}$. Following, the transfection mixture was replaced with fresh medium to culture for $48 \mathrm{~h}$. Finally, the A549 cells were assayed using the appropriate protocol.

Reverse transcription-quantitative polymerase chain reaction $(R T-q P C R)$. RT-qPCR was selected to determine the expression of HDAC5 mRNA. The primers for HDAC5 (GenBank: BC051824.1) were: Left primer gtgacaccgtgtggaatgag and right primer agtccacgatgaggaccttg. The primers for $\beta$-actin (GenBank: M10277.1) were: Left primer ctcttccagcettccttcct and right primer agcactgtgttggegtacag. Total RNA was extracted using Trizol reagent obtained from Beyotime Institute of Biotechnology (cat. no. R0016). Then mRNAs were reverse transcribed into cDNA using BeyoRT ${ }^{\mathrm{TM}}$ cDNA Synthesis Kit (Beyotime Institute of Biotechnology; cat. no. D7166). qPCR analyses were performed using the BeyoFast $^{\mathrm{TM}}$ SYBR Green qPCR Mix (Beyotime Institute of Biotechnology; cat. no. D7260) on Applied Biosystems ${ }^{\circledR}$ 7500 Real-Time PCR Systems (Thermo Fisher Scientific, Inc.). The following thermocycling conditions were used for qPCR. Firstly $95^{\circ} \mathrm{C}$ for $5 \mathrm{~min}$, followed by 36 cycles of $95^{\circ} \mathrm{C}$ for $10 \mathrm{sec}, 60^{\circ} \mathrm{C}$ for $30 \mathrm{sec}$, and $72^{\circ} \mathrm{C} 30 \mathrm{sec}$. The genes $\beta$-actin was used as internal control for RT-qPCR. All RT-PCRs were 
A

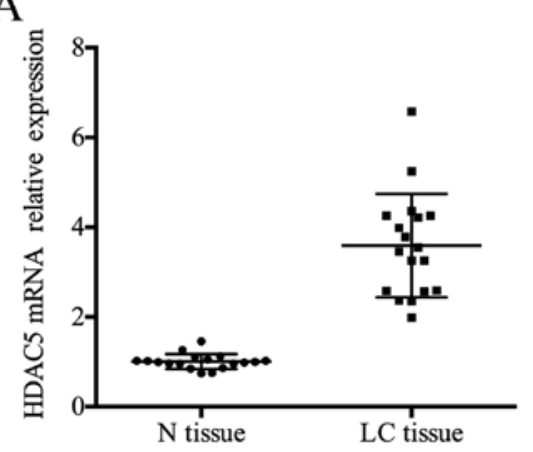

$\mathrm{B}$
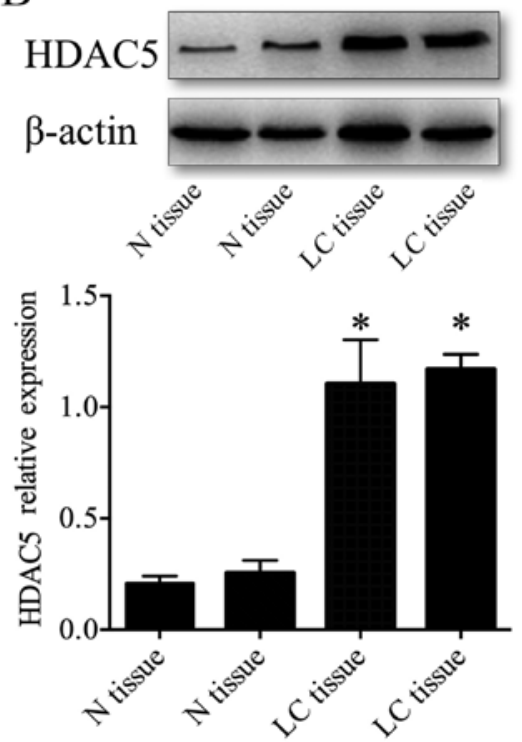

$\mathrm{C}$

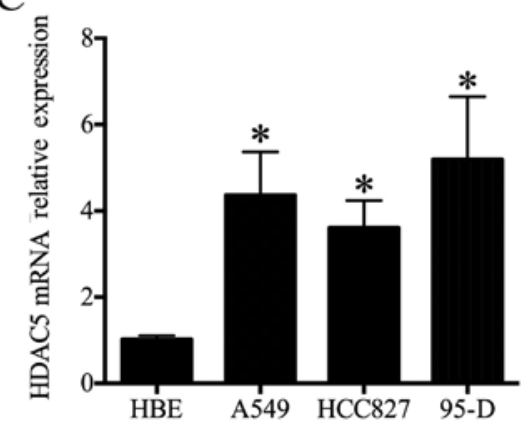

$\mathrm{D}$
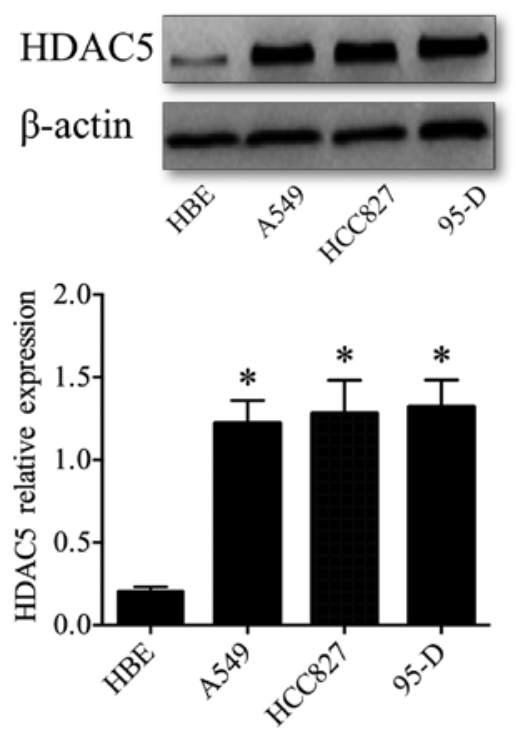

Figure 1. HDAC5 is significantly upregulated in lung cancer tissues and cell lines. (A and B) Elevated expression of HDAC5 was noted in the LC (lung cancer) tissues. "P $<0.05$ vs. $\mathrm{N}$ tissues (tumor-adjacent normal tissues). (C and D) Elevated expression of HDAC5 was noted in $3 \mathrm{LC}$ cell lines. "P $<0.05$ vs. HBE cells. HDAC5, histone deacetylase 5 .

performed in triplicate and the relative fold differences in gene expression were calculated according to the $2^{-\Delta \Delta \mathrm{Ct}}$ method.

Western blot analysis. Total proteins were extracted from lung cancer tissues, lung cancer-adjacent normal tissues, lung cancer cell lines (A549, HCC827 and 95-D) and HBE cells using a protein extraction kit, and were quantified using a BCA protein concentration assay kit. Proteins were separated by $12 \%$ SDS-polyacrylamide gel electrophoresis and transferred to a PVDF membrane using a wet-type transblotting apparatus (Bio-Rad Laboratories, Richmond, CA, USA). Then the PVDF membrane was blocked with $5 \%$ skimmed milk diluted in TBS (10 mM Tris- $\mathrm{HCl}, \mathrm{pH} 7.5,150 \mathrm{mM} \mathrm{NaCl})$ solution for $1 \mathrm{~h}$, and incubated with the primary antibody at $4^{\circ} \mathrm{C}$ overnight. All primary antibodies were diluted 1:2,000 in TBST buffer (10 mM Tris- $\mathrm{HCl}, \mathrm{pH} 7.5,150 \mathrm{mM} \mathrm{NaCl}$ and $0.1 \%$ Tween-20) supplemented with 5\% non-fat milk. Next morning, the PVDF membrane was washed $3 \times 5 \mathrm{~min}$ in TBST and incubated with the corresponding secondary antibody at room temperature for $2 \mathrm{~h}$. All secondary antibodies were diluted 1:5,000 in TBST buffer. Following, the PVDF membrane was washed $3 \times 5$ min in TBST and bands were visualized using the ECL chemiluminescence reagent. The relative expression of the target protein was valuated with the gray value ratio of target protein content to $\beta$-actin (target protein $/ \beta$-actin) content by Quantity One software (Bio-Rad Laboratories, Hercules, CA, USA).

MTT assay. MTT assay was used to examine the effects of HDAC5 on the viability of A549 cells. Briefly, A549 cells were seeded in 96-well plates and allowed to adhere overnight. Then, A549 cells were incubated with $10 \mu \mathrm{l}$ of MTT $(5 \mathrm{mg} / \mathrm{ml})$ for $4 \mathrm{~h}$. The mixture culture medium was replaced by $150 \mu \mathrm{l}$ of dimethyl sulfoxide (DMSO) to dissolve the crystals. The optical density (OD) values at $570 \mathrm{~nm}$ (test wavelength) and $630 \mathrm{~nm}$ (reference wavelength) were examined on a 96-well micro test spectrophotometer (BioTek Instruments, Inc., Winooski, VT, USA). The relative cell viability was calculated by the equation as described in a previous study (19) and the experiments were run in triplicate.

Flow cytometric analysis. The effects of HDAC5 on the A549 cell cycle and apoptosis were determined using flow cytometry. First, A549 cells were seeded in serum-free RPMI-1640 medium for $24 \mathrm{~h}$ to synchronize and then incubated with complete RPMI-1640 medium for 24 h. Following, A549 cells were trypsinized, washed, harvested, fixed with $70 \%$ ice-cold 
ethanol and stored at $-20^{\circ} \mathrm{C}$. On the next day, A549 cells were washed with citrate phosphate buffer and PBS in turn, treated with PBS containing $100 \mu \mathrm{g} / \mathrm{ml}$ of RNase A at $37^{\circ} \mathrm{C}$ for $30 \mathrm{~min}$, and then cultured in PBS containing $100 \mu \mathrm{g} / \mathrm{ml}$ of propidium iodide (PI) at room temperature for $30 \mathrm{~min}$. Finally, cell cycle distribution was determined using flow cytometry (BD Biosciences, Franklin Lakes, NJ, USA). The experiments were performed in triplicate.

A549 cell apoptosis was quantitated by staining with Annexin V-FITC. Briefly, A549 cells were washed, collected, and resuspended in $195 \mu \mathrm{l}$ of Annexin V-FITC binding buffer. Following, $5 \mu \mathrm{l}$ of Annexin V-FITC was added into the Annexin V-FITC binding buffer and incubated in the dark at room temperature for $10 \mathrm{~min}$. Then, A549 cells were collected by centrifugation for $5 \mathrm{~min}$ at $1,500 \mathrm{xg}$, and gently resuspended in $190 \mu \mathrm{l}$ of Annexin V-FITC binding buffer. Finally, $10 \mu \mathrm{l}$ of PI staining solution was added into the Annexin V-FITC binding buffer and kept on ice in the dark until flow cytometric analysis. CellQuest software (BD Biosciences) was used to analyze the datum, and the analysis was run in triplicate.

Transwell invasion analysis. Transwell invasion chamber was used to evaluate the invasive ability of the A549 cells. Briefly, the chamber filter was washed with serum-free RPMI-1640 medium, and then the upper side of the filter was evenly covered with $20 \mu 1$ of Matrigel (1:2 dilution with RPMI-1640). The chamber was divided by two compartments including the supper chamber and the lower chamber. For invasion assays, $200 \mu \mathrm{l}$ of serum-free RPMI-1640 medium containing $1 \times 10^{5}$ A549 cells were added in the upper chamber of the Transwell invasion system, while $500 \mu 1$ of RPMI-1640 medium containing $10 \%$ FBS were added into the lower chamber. Then the Transwell invasion system was incubated for $48 \mathrm{~h}$ in an incubator. Following, the cells on the upper surface of the filter were removed with a sterile cotton swab. Those cells that invaded to the lower surface of the filter and invaded in the lower chamber were collected and assessed by MTT assay. The results are presented as the mean $\pm \mathrm{SD}$, and the experiment was performed in triplicate.

Statistical analysis. All data are expressed as mean \pm SD from at least three independent experiments. SPSS 17.0 software (SPSS, Inc., Chicago, IL, USA) was used to analyze the experimental data with Student's t-test or one-way ANOVA followed by Tukey's post hoc test. The results were considered statistically significant at $\mathrm{P}<0.05$. GraphPad Prism software version 5.0 (GraphPad Software, Inc., San Diego, CA, USA) was applied to draw the graphs.

\section{Results}

Overexpression of HDAC5 in lung cancer tissues and cell lines. We first examined HDAC5 protein and mRNA levels in human lung cancer tissues by western blotting and RT-qPCR. The results showed that the expression of HDAC5 protein and mRNA was significantly upregulated in lung cancer tissues compared to that observed in the lung cancer-adjacent normal tissues $(\mathrm{P}<0.05)$ (Fig. 1A and $\mathrm{B})$. The expression profile of HDAC5 protein and mRNA in lung cancer tissues were similar to those in lung cancer cell lines, which showed that

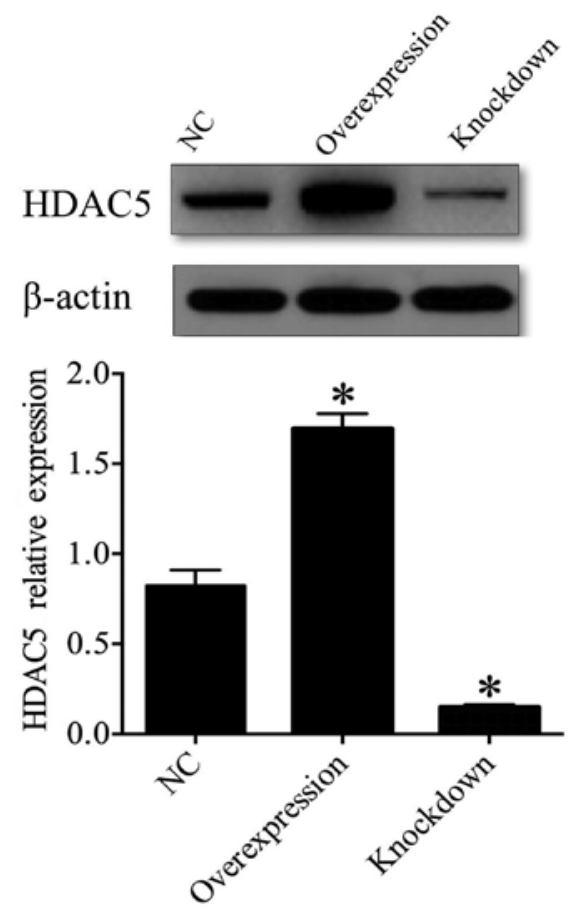

Figure 2. Expression of HDAC5 in lung cancer A549 cell lines. HDAC5 displayed a significant upregulation in the A549 cells transfected with pcDNA3.1-HDAC5 (overexpression) and showed an obvious downregulation in the A549 cells transfected with HDAC5 siRNA (knockdown) compared with the A549 cells transfected with the vector only (NC). " $\mathrm{P}<0.05$ vs. NC. HDAC5, histone deacetylase 5 .

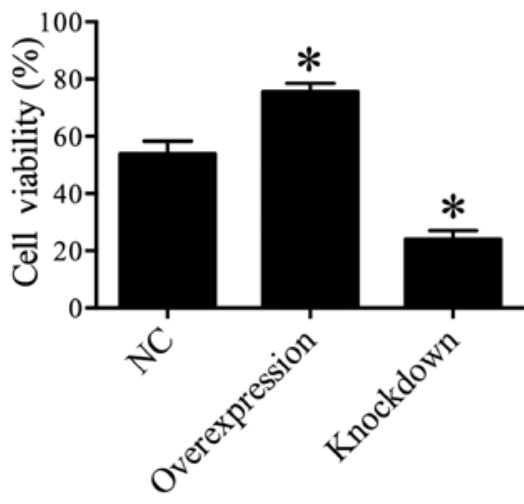

Figure 3.Effect of HDAC5 on A549 cell viability.Histogram is shown comparing the cell viability among the A549 cells transfected with pcDNA3.1-HDAC5 (overexpression) and HDAC5 siRNA (knockdown) compared with the A549 cells transfected with the vector only $(\mathrm{NC}) .{ }^{*} \mathrm{P}<0.05$ compared with NC. All experiments were run in triple. HDAC5, histone deacetylase 5.

HDAC5 protein and mRNA displayed significant upregulation in human lung cancer cell lines (A549, HCC827 and 95-D) compared to that observed in the human bronchial epithelial (HBE) cells $(\mathrm{P}<0.05)$ (Fig. 1C and D). These data demonstrated that the elevated HDAC5 may be important in the tumorigenesis and progression of lung cancer.

In order to address the function of HDAC5 in the tumorigenesis and progression of lung cancer, A549 cells were chosen for further investigation unless specified otherwise. We generated A549 cells in which HDAC5 was either overexpressed or depleted. Western blot analysis indicated that the expression of HDAC5 protein was significantly 

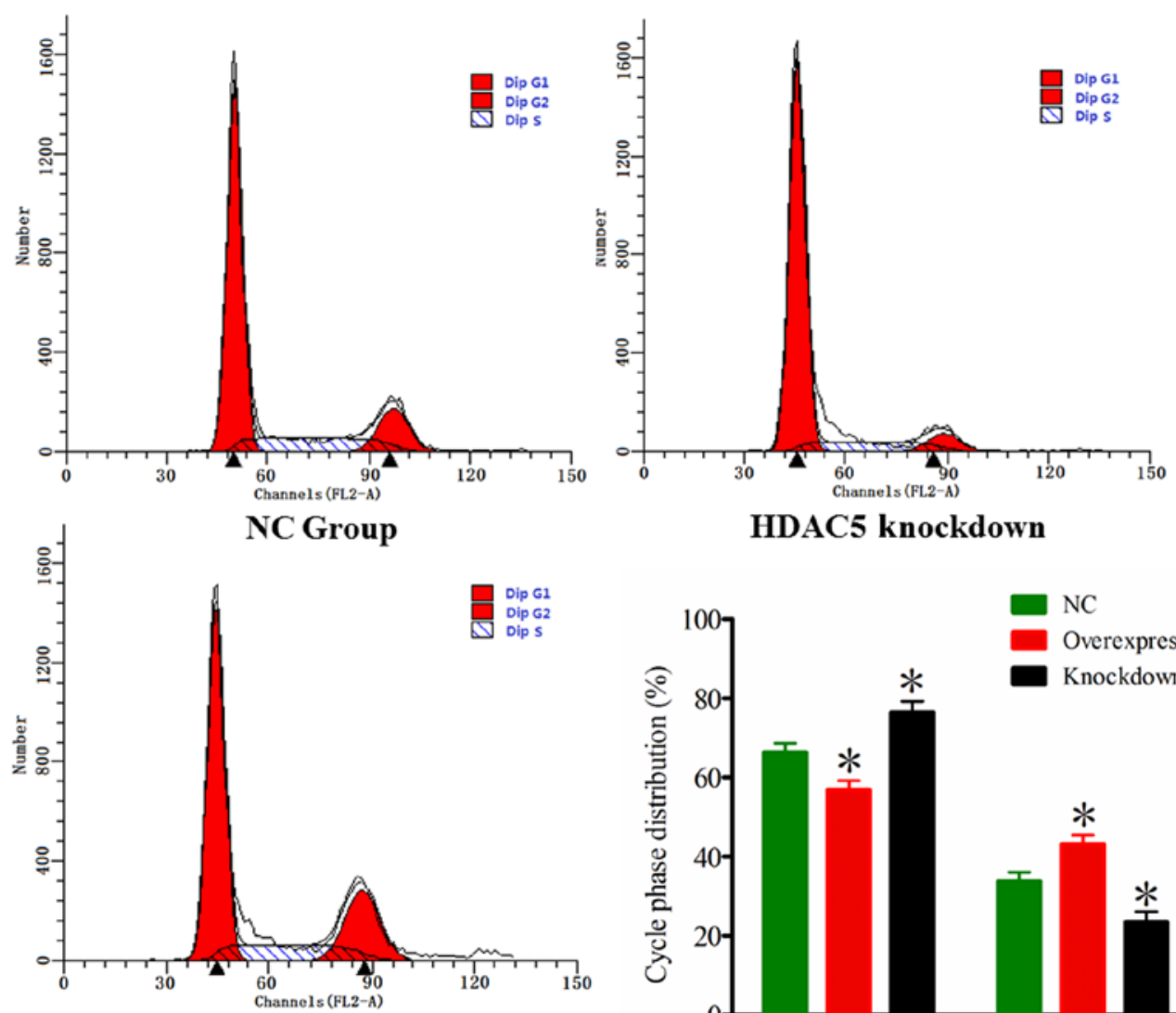

HDAC5 knockdown

HDAC5 overexpression

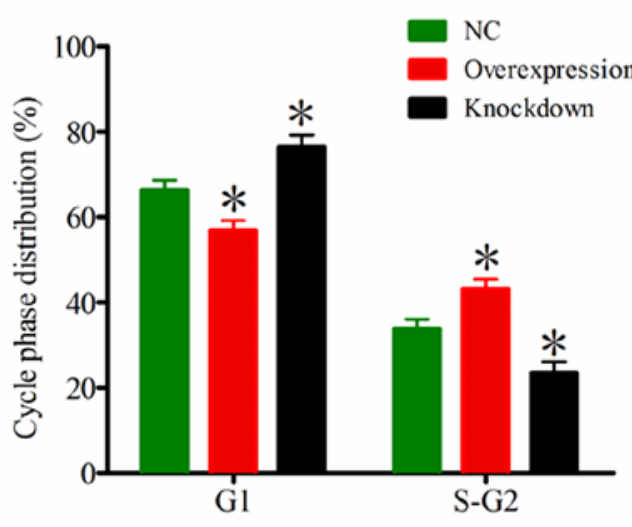

Figure 4. Effect of HDAC5 on A549 cell cycle distribution. Histogram (lower right) is shown comparing the cell cycle distribution among the A549 cells transfected with pcDNA3.1-HDAC5 (overexpression) and HDAC5 siRNA (knockdown) compared with the A549 cells transfected with the vector only (NC) The percentage of A549 cells in each phase of the cell cycle is expressed as the mean $\pm \mathrm{SD}$. ${ }^{*} \mathrm{P}<0.05$ compared with NC. The other images are representative FCM images. All experiments were run in triple. HDAC5, histone deacetylase 5.

upregulated in the HDAC5 overexpression group (transfected with pcDNA3.1-HDAC5) $(\mathrm{P}<0.05)$, and was obviously downregulated in the HDAC5-knockdown group (transfected with HDAC5 siRNA) compared to the control group (NC, transfected with vector only) $(\mathrm{P}<0.05)$ (Fig. 2), which suggested that A549 cell models, in which HDAC5 was either overexpressed or depleted, were successfully established.

HDAC5 enhances A549 cell viability. In order to explore the effect of HDAC5 on the cell viability of lung cancer cells, MTT assay was performed and the results suggested that A549 cell viability was significantly enhanced in the HDAC5 overexpression group compared with the control group $(\mathrm{P}<0.05)$, while the cell viability of A549 cells was markedly inhibited in the HDAC5 knockdown group compared with the control group $(\mathrm{P}<0.05)$ (Fig. 3). These data indicated that elevated HDAC5 may play a crucial role in the increase in A549 cell viability.

HDAC5 promotes A549 cell cycle progression. In order to address whether HDAC5 is associated with A549 cell cycle progression, we evaluated the cell cycle distribution of A549 cells by FCM. The results indicated that there were more A549 cells in the $\mathrm{S}$ and $\mathrm{G} 2$ phases, and less A549 cells in the G1 phase in the HDAC5 overexpression group compared to these populations in the control group $(\mathrm{P}<0.05)$. On the

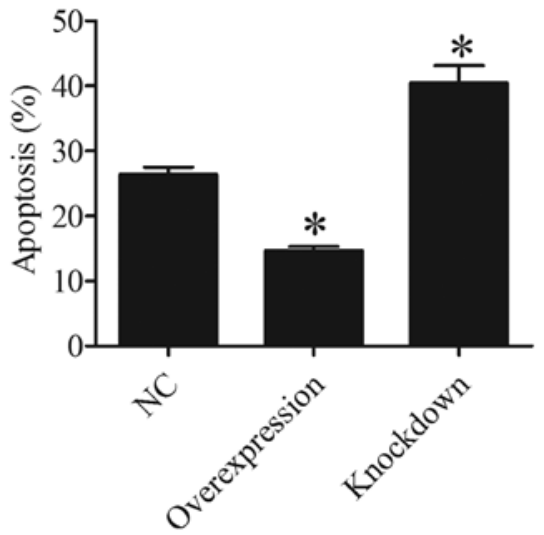

Figure 5. Effect of HDAC5 on A549 cell apoptosis. Histogram is shown comparing the cell apoptosis among the A549 cells transfected with pcDNA3.1-HDAC5 (overexpression) and HDAC5 siRNA (knockdown) compared with the A549 cells transfected with the vector only (NC). The percentage of apoptotic A549 cells is expressed as the mean $\pm \mathrm{SD}$. ${ }^{*} \mathrm{P}<0.05$ compared with NC. All experiments were run in triple. HDAC5, histone deacetylase 5; SD, standard deviation.

contrary, there were less A549 cells in the S and G2 phases, and more A549 cells in the G1 phase in the HDAC5 knockdown group compared to these populations in the control group $(\mathrm{P}<0.05)$ (Fig. 4). These data demonstrated that HDAC5 may promote A549 cell cycle progression. 


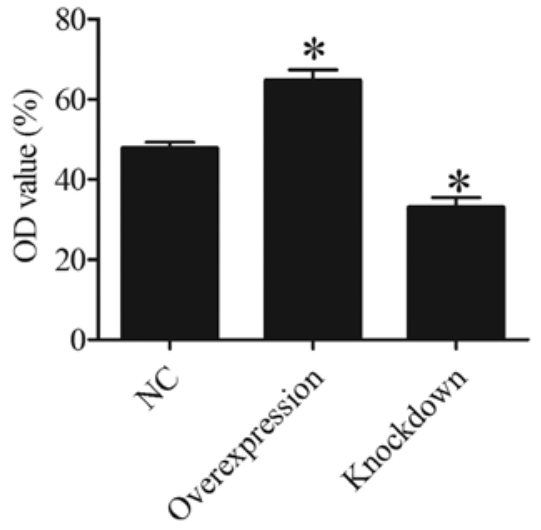

Figure 6. Effect of HDAC5 on A549 cell invasion. Histogram is shown comparing the OD value of invaded A549 cells transfected with pcDNA3.1-HDAC5 (overexpression) and HDAC5 siRNA (knockdown) compared with the A549 cells transfected with the vector only (NC). The OD value of invaded A549 cells is expressed as the mean \pm SD. ${ }^{*} \mathrm{P}<0.05$ compared with NC. All experiments were run in triple. HDAC5, histone deacetylase 5; SD, standard deviation; OD, optical density.

HDAC5 inhibits A549 cell apoptosis. In order to ascertain whether HDAC5 is associated with A549 cell apoptosis, we evaluated the cell apoptosis of A549 cells by FCM. The results showed that there were less apoptotic A549 cells in the HDAC5 overexpression group than that in the control group $(\mathrm{P}<0.05)$. On the contrary, more apoptotic A549 cells were found in the HDAC5 knockdown group compared to that in the control group $(\mathrm{P}<0.05)$ (Fig. 5). These data suggested that HDAC5 plays a crucial role in the inhibition of A549 cell apoptosis.

HDAC5 enhances the invasive ability of A549 cells. To determine whether HDAC5 is associated with A549 cell invasion, Transwell invasion assay was used to evaluate the effect of HDAC5 on A549 cells. The results suggested that the OD value of the invaded A549 cells in the HDAC5 overexpression group was higher than that in the control group $(\mathrm{P}<0.05)$. On the contrary, a lower OD value was found in the HDAC5 knockdown group compared to that noted in the control group $(\mathrm{P}<0.05)$ (Fig. 6). These data demonstrated that more A549 cells invaded through the polycarbonate membrane in HDAC5 overexpression group, and less A549 cells invaded through the polycarbonate membrane in HDAC5 knockdown group compared to control group, which indicated that HDAC5 strengthen the invasive ability of A549 cells.

HDAC5 increases the expression of DLL4, Sixl, Notch 1 and Twist 1. HDAC5 has fundamental roles in the epigenetic regulation of gene expression and contributes to proliferation, differentiation, apoptosis, cell cycle and invasion (8-10). Previous studies suggest that HDAC5 is an important regulatory factor for the expression of DLL4, Six1, Notch 1 and Twist $1(8,9,16,20)$. Therefore, the expression of DLL4, Six1, Notch 1 and Twist 1 was detected in this study, and the results indicated that the expression of DLL4, Six 1, Notch 1 and Twist 1 was significantly enhanced in the HDAC5 overexpression group, and obviously suppressed in the HDAC5 knockdown group compared with the control group $(\mathrm{P}<0.05)$ (Fig. 7). These results suggested that HDAC5 increased the expression of DLL4, Six1, Notch 1 and Twist 1 in the A549 cells.

\section{Discussion}

Lung cancer is one of the most common malignant neoplasms, as well as the most common cause of cancer-related mortality. Most lung cancers are squamous cell carcinomas, small cell carcinomas or adenocarcinomas (21). The prognosis of patients with lung cancer is still less than satisfactory, and the 5 -year survival rate does not exceed $15 \%$ (2-5). This is mainly due to the fact that many key factors regulating the malignant phenotype of lung cancer have not been studied clearly. HDAC5 is frequently dysregulated in human malignancies and has therefore become a therapeutic target in cancer therapy $(8,9,11,16,20)$. However, whether HDAC5 is involved in lung cancer incidence, migration and invasion remains elusive.

In the present study, our data showed that HDAC5 displayed significantly high expression in lung cancer tissues and cell lines. The expression profile of HDAC5 in lung cancer was consistent with that in breast cancer, colorectal cancer and glioma $(8,15,22)$. Elevated HDAC5 was found to promote the proliferation of colorectal cancer cells through upregulation of DLL4 (8). HDAC5 was found to be increased in human glioma tissues and to promote the proliferation of glioma cells by the
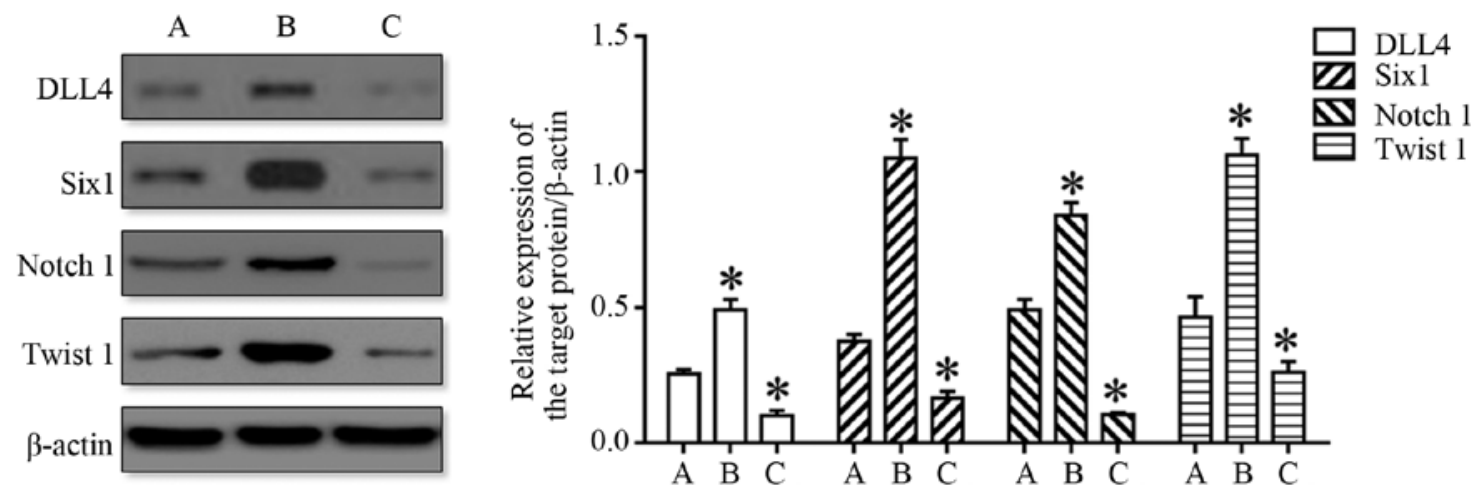

Figure 7. HDAC5 increases the expression of DLL4, Six1, Notch 1 and Twist 1 in A549 cells. The image on the left displays the representative western blot image. The histogram shown compares the relative expression levels of DLL4, Six1, Notch 1 and Twist 1 in the A549 cells transfected with pcDNA3.1-HDAC5 (overexpression) and HDAC5 siRNA (knockdown) compared with the A549 cells transfected with the vector only (NC). * $<0.05$ vs. A. (A, NC, B, Overexpression, C, Knockdown). HDAC5, histone deacetylase 5. 


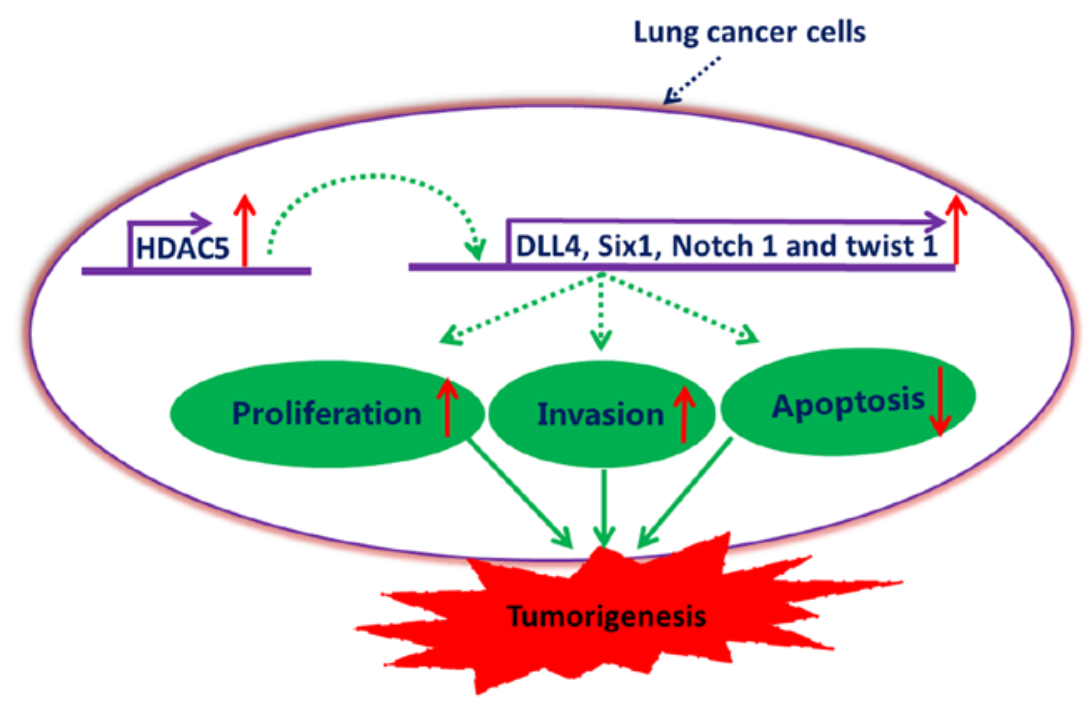

Figure 8. A model for the role of HDAC5 in the tumorigenesis of lung cancer. The integrated activation of DLL4, Six1, Notch 1 and Twist 1 by HDAC5 results in the tumorigenesis of lung cancer. HDAC5, histone deacetylase 5.

upregulation of Notch 1 (20). These data indicate that elevated HDAC5 may play a central role in the tumorigenesis of lung cancer.

In order to elucidate the role of HDAC5 in lung cancer cells, A549 cell models, in which HDAC5 was either overexpressed or depleted, were generated. HDAC5 displayed a higher expression in the HDAC5 overexpression group and a lower expression in the HDAC5 knockdown group compared to the control group, which indicated that the A549 cell models were successfully established. In view of the established lung cancer A549 cell models, the effects of HDAC5 on cell viability, cell cycle distribution, apoptosis and invasion of A549 cells were determined, and the results showed that HDAC 5 overexpression enhanced the cell viability and proliferation of A549 cells. On the contrary, HDAC5 inhibition suppressed the cell viability and proliferation of A549 cells, leading to cell growth inhibition and cell cycle G1 phase arrest in A549 cells. These data indicated that HDAC5 improved the cell growth and proliferation of A549 cells. The effect on the proliferation of A549 cells was consistent with that of other cancers reported by previous studies which demonstrated that elevated HDAC5 promoted the proliferation of colorectal cancer cells, hepatocellular carcinoma cells and glioma cells, while downregulation of HDAC5 caused a significant inhibition of colorectal cancer cell, hepatocellular carcinoma cell and glioma cell proliferation $(8,16,20)$. The results also showed that overexpression of HDAC5 displayed an obvious inhibition of A549 cell apoptosis, and the inhibition of HDAC5 facilitated A549 cell apoptosis, which indicated that HDAC5 inhibited A549 cell apoptosis. The results in this study were also confirmed by previous studies concerning breast cancer and hepatocellular carcinoma, which reported that knockdown of HDAC5 reduced tumorigenesis and enhanced apoptosis $(15,22-24)$. HDAC5 was extensively expressed in many human cancers. HDAC5, overexpressed in neuroblastoma, was found to trigger neuroblastoma cell invasion and metastasis (25), and knockdown of HDAC5 restrained breast cancer cell proliferation, invasion and metastasis (15). Furthermore, the effect of HDAC5 on A549 cell invasion was evaluated by
Transwell invasion assay. The results revealed that overexpression of HDAC5 was associated with the increased invasive capacity of A549 cells, and the inhibition of HDAC5 was associated with the decreased invasive capacity of A549 cells.

In summary, these results demonstrated that HDAC5 was associated with increased A549 cell growth, proliferation, and invasion and decreased A549 cell apoptosis. Nevertheless, the detailed mechanism or the downstream HDAC5 targets in human lung cancer cells remains unclear. HDAC5 promoted colorectal cancer cell proliferation by upregulating DLL4 expression (8), glioma cell proliferation by upregulation of Notch 1 (20), human hepatocellular carcinoma cell proliferation by upregulating Six 1 expression (16), and osteosarcoma progression by upregulation of Twist 1 expression (9). Therefore, the expression levels of DLL4, Six1, Notch 1 and Twist 1 were also determined in this study, and the results indicated that HDAC5 increased the expression of DLL4, Six 1, Notch 1 and Twist 1 in the A549 cells. Our results were in line with the results from previous studies that found that overexpression of DLL4 was associated with poor outcomes of patients with pancreatic adenocarcinoma, and elevated DLL4 promoted renal carcinoma cell metastasis $(26,27)$. Thus, we may speculate that elevated HDAC5 promotes the expression of DLL4 in lung cancer, and contributes to poor patient outcomes and metastasis. Increased Six1 was found to be associated with the poor prognosis of prostate cancer patients and enhanced pancreatic cancer cell proliferation through upregulation of cyclin D1 $(28,29)$. Conversely, downregulation of Six 1 suppressed colorectal cancer cell growth and invasion (30). From our results, we could infer that elevated HDAC5 could promote the expression of Six 1 in lung cancer, and contribute to lung cancer cell proliferation and poor prognosis. Notch 1 signaling, activated in many cancers, promoted the malignant features including epithelial to mesenchymal transition of cancers through NF- $\mathrm{BB}$ activation $(31,32)$. Notably, DLL4-Notch signaling was found to participate in the formation of large vessels in tumors, leading to distant metastasis of tumors (33). Inhibition of Notch 1 signaling pathway was found to inhibit breast cancer cell 
proliferation and invasion (34). Twist 1, a key factor in the promotion of metastasis of cancer cells, promoted cell growth and metastasis in acute myeloid leukemia $(35,36)$. In view of these data, we may conjecture that elevated HDAC5 in lung cancer could promote the expression of Notch 1 and Twist 1 , and then promote EMT and distant metastasis. HDAC5 could repress the expression of miR-125a-5p in human breast cancer (22). Our previous study showed that miR-125a-5p was downregulated and acted as a tumor suppressor in lung carcinoma by directly targeting STAT3 (37). These data may be responsible for the explanation that HDAC5 promoted A549 cell growth, proliferation, invasion and inhibited A549 cell apoptosis.

In summary, our data demonstrated that HDAC5 was significantly upregulated in lung cancer, and elevated HDAC5 may be involved in the potentiation of proliferation and invasion of lung cancer cells, as well as the inhibition of lung cancer cell apoptosis, at least partially, by the upregulation of DLL4, Six1, Notch 1 and Twist 1 (Fig. 8). This study provides evidence for the potential application of HDAC5 inhibitors in the therapy of lung cancer.

\section{Acknowledgements}

We thank Dr Hualin Sun for his technical support.

\section{Funding}

The present study was supported by the National Natural Science Foundation of China (grant no. 81501967).

\section{Availability of data and materials}

The datasets used during the present study are available from the corresponding author upon reasonable request.

\section{Authors' contributions}

LZ and JHS conceived and designed the study. LZ, SYS, SMY, MMG and XH performed the experiments. LZ wrote the paper. LZ, SYS, SMY, MMM, XH and JHS reviewed and edited the manuscript. All authors read and approved the manuscript and agree to be accountable for all aspects of the research in ensuring that the accuracy or integrity of any part of the work are appropriately investigated and resolved.

\section{Ethics approval and consent to participate}

The present study was reviewed and approved by the Ethics Committee of the Affiliated Hospital of Nantong University. All patients volunteered to participate in the study and signed a written informed consent.

\section{Patient consent for publication}

Not applicable.

\section{Competing interests}

The authors declare that they have no competing interests.

\section{References}

1. Denton EJ, Hart D, Wainer Z, Wright G, Russell PA and Conron M: Changing trends in diagnosis, staging, treatment and survival in lung cancer: Comparison of three consecutive cohorts in an Australian lung cancer centre. Intern Med J 46: 946-954, 2016.

2. Osińska I and Domagała-Kulawik J: Bronchoalveolar lavage in lung cancer-diagnostic value and assessment of the anti-cancer immune response. Postepy Hig Med Dosw 67: 1119-1127, 2013 (In Polish).

3. Giangreco A, Groot KR and Janes SM: Lung cancer and lung stem cells: Strange bedfellows? Am J Respir Crit Care Med 175: 547-553, 2007.

4. Grivaux M, Debieuvre D, Herman D, Lemonnier C, Marcos JM, Crequit J, Vuillermoz-Blas S, Barre P, Saillour M and Martin F: Early mortality in lung cancer: French prospective multicentre observational study. BMC Pulm Med 16: 45, 2016.

5. Whiteside TL: Stimulatory role of exosomes in the context of therapeutic anti-cancer vaccines. Biotarget 1: 5, 2017.

6. Al-Mulla F, Bitar MS, Al-Maghrebi M, Behbehani AI, Al-Ali W, Rath O, Doyle B, Tan KY, Pitt A and Kolch W: Raf kinase inhibitor protein RKIP enhances signaling by glycogen synthase kinase-3 $\beta$. Cancer Res 71: 1334-1343, 2011.

7. Noma D, Inamura K, Matsuura Y, Ninomiya H, Ichinose J, Nakao M, Mun M, Ishikawa Y and Okumura S: ALK-rearranged lung adenocarcinoma showing intra-bronchial protrusion: A case of actually peripheral origin with a rare spreading pattern. Biotarget 1: 15, 2017.

8. He P, Liang J, Shao T, Guo Y, Hou Y and Li Y: HDAC5 promotes colorectal cancer cell proliferation by up-regulating DLL4 expression. Int J Clin Exp Med 8: 6510-6516, 2015.

9. Chen J, Xia J, Yu YL, Wang SQ, Wei YB, Chen FY, Huang GY and Shi JS: HDAC5 promotes osteosarcoma progression by upregulation of Twist 1 expression. Tumour Biol 35: 1383-1387, 2014.

10. Fang Q, Xu T, Wu C, Zhou S and Sun H: Biotargets in neural regeneration. Biotarget 1: 6, 2017.

11. Xiao H, Jiao J, Wang L, O'Brien S, Newick K, Wang LC, Falkensammer E, Liu Y, Han R, Kapoor V, et al: HDAC5 controls the functions of Foxp3 ${ }^{+}$T-regulatory and $\mathrm{CD}^{+} \mathrm{T}$ cells Int J Cancer 138: 2477-2486, 2016.

12. Guise AJ and Cristea IM: Approaches for studying the subcellular localization, interactions, and regulation of histone deacetylase 5 (HDAC5). Methods Mol Biol 1436: 47-84, 2016.

13. Greco TM, Yu F, Guise AJ and Cristea IM: Nuclear import of histone deacetylase 5 by requisite nuclear localization signal phosphorylation. Mol Cell Proteomics 10: M110 004317, 2011.

14. Lin M, Zhu Q, Wang J, Yang W, Fan H, Yi J and Jiang M: Molecules involved in acrosomal exocytosis and cortical granule exocytosis. Biotarget 1: 11, 2017.

15. Li A, Liu Z, Li M, Zhou S, Xu Y, Xiao Y and Yang W: HDAC5, a potential therapeutic target and prognostic biomarker, promotes proliferation, invasion and migration in human breast cancer. Oncotarget 7: 37966-37978, 2016.

16. Feng GW, Dong LD, Shang WJ, Pang XL, Li JF, Liu L and Wang Y: HDAC5 promotes cell proliferation in human hepatocellular carcinoma by up-regulating Six1 expression. Eur Rev Med Pharmacol Sci 18: 811-816, 2014.

17. Liu J, Gu J, Feng Z, Yang Y, Zhu N, Lu W and Qi F: Both HDAC5 and HDAC6 are required for the proliferation and metastasis of melanoma cells. J Transl Med 14: 7, 2016.

18. Milde T, Oehme I, Korshunov A, Kopp-Schneider A Remke M, Northcott P, Deubzer HE, Lodrini M, Taylor MD, von Deimling A, et al: HDAC5 and HDAC9 in medulloblastoma: Novel markers for risk stratification and role in tumor cell growth. Clin Cancer Res 16: 3240-3252, 2010.

19. Wang HJ, Ruan HJ, He XJ, Ma YY, Jiang XT, Xia YJ, Ye ZY and Tao HQ: MicroRNA-101 is down-regulated in gastric cancer and involved in cell migration and invasion. Eur J Cancer 46: 2295-2303, 2010.

20. Liu Q, Zheng JM, Chen JK, Yan XL, Chen HM, Nong WX and Huang HQ: Histone deacetylase 5 promotes the proliferation of glioma cells by upregulation of Notch 1. Mol Med Rep 10: 2045-2050, 2014.

21. Medenica M, Medenica M, Bojović O, Soldatović I and Durutović I: Changing trends in incidence of lung cancer by histological type in Montenegro. Srp Arh Celok Lek 142: 23-28, 2014. 
22. Hsieh TH, Hsu CY, Tsai CF, Long CY, Wu CH, Wu DC, Lee JN, Chang WC and Tsai EM: HDAC inhibitors target HDAC5, upregulate microRNA-125a-5p, and induce apoptosis in breast cancer cells. Mol Ther 23: 656-666, 2015.

23. Zhang M, Pan Y, Dorfman RG, Czen Z, Liu F, Zhou Q, Huang S, Zhang J, Yang D and Liu J: AR-42 induces apoptosis in human hepatocellular carcinoma cells via HDAC5 inhibition. Oncotarget 7: 22285-22294, 2016.

24. Fan J, Lou B, Chen W, Zhang J, Lin S, Lv FF and Chen Y: Down-regulation of HDAC5 inhibits growth of human hepatocellular carcinoma by induction of apoptosis and cell cycle arrest. Tumour Biol 35: 11523-11532, 2014.

25. Fabian J, Opitz D, Althoff K, Lodrini M, Hero B, Volland R, Beckers A, de Preter K, Decock A, Patil N, et al: MYCN and HDAC5 transcriptionally repress CD9 to trigger invasion and metastasis in neuroblastoma. Oncotarget 7: 66344-66359, 2016.

26. Zhou L, Yu L, Ding G, Chen W, Zheng S and Cao L: Overexpressions of DLL4 and CD105 are associated with poor prognosis of patients with pancreatic ductal adenocarcinoma. Pathol Oncol Res 21: 1141-1147, 2015.

27. Huang QB, Ma X, Li HZ, Ai Q, Liu SW, Zhang Y, Gao Y, Fan Y, Ni D, Wang BJ and Zhang X: Endothelial Delta-like 4 (DLL4) promotes renal cell carcinoma hematogenous metastasis. Oncotarget 5: 3066-3075, 2014.

28. Zeng J, Shi R, Cai CX, Liu XR, Song YB, Wei M and Ma WL: Increased expression of Six 1 correlates with progression and prognosis of prostate cancer. Cancer Cell Int 15: 63, 2015.

29. Li Z, Tian T, Lv F, Chang Y, Wang X, Zhang L, Li X, Li L, Ma W, Wu J and Zhang M: Six1 promotes proliferation of pancreatic cancer cells via upregulation of cyclin D1 expression. PLoS One 8: e59203, 2013.
30. Li Z, Tian T, Hu X, Zhang X, Li L, Nan F, Chang Y, Wang X, Sun Z, Lv F, et al: Targeting Six1 by lentivirus-mediated RNA interference inhibits colorectal cancer cell growth and invasion. Int J Clin Exp Pathol 7: 631-639, 2014.

31. Fender AW, Nutter JM, Fitzgerald TL, Bertrand FE and Sigounas G: Notch-1 promotes stemness and epithelial to mesenchymal transition in colorectal cancer. J Cell Biochem 116: 2517-2527, 2015

32. Li L, Zhao F, Lu J, Li T, Yang H, Wu C and Liu Y: Notch-1 signaling promotes the malignant features of human breast cancer through NF- $\kappa$ B activation. PLoS One 9: e95912, 2014

33. Li JL, Sainson RC, Oon CE, Turley H, Leek R, Sheldon H, Bridges E, Shi W, Snell C, Bowden ET, et al: DLL4-Notch signaling mediates tumor resistance to anti-VEGF therapy in vivo. Cancer Res 71: 6073-6083, 2011.

34. Zhang Q, Yuan Y, Cui J, Xiao T and Jiang D: Paeoniflorin inhibits proliferation and invasion of breast cancer cells through suppressing Notch-1 signaling pathway. Biomed Pharmacother 78: 197-203, 2016.

35. Wushou A, Hou J, Zhao YJ and Shao ZM: Twist-1 up-regulation in carcinoma correlates to poor survival. Int J Mol Sci 15: 21621-21630, 2014.

36. Wang N, Guo D, Zhao YY, Dong CY, Liu XY, Yang BX, Wang SW, Wang L, Liu QG, Ren Q, et al: TWIST-1 promotes cell growth, drug resistance and progenitor clonogenic capacities in myeloid leukemia and is a novel poor prognostic factor in acute myeloid leukemia. Oncotarget 6: 20977-20992, 2015.

37. Zhong L, Sun S, Shi J, Cao F, Han X and Chen Z: MicroRNA-125a-5p plays a role as a tumor suppressor in lung carcinoma cells by directly targeting STAT3. Tumour Biol 39: 1010428317697579, 2017. doi: 10.1177/1010428317697579. 